\title{
EFEITO DE DINITRAMINE, APLICADO EM PRÉ-PLANTIO INCORPORADO, EM CULTURA DE SOJA (Glycine $\max (L$.) Merril)
}

\begin{abstract}
RESUMO
Com a finalidade de se obter maiores conhecimentos sobre a ação do herbicida dinitramine no controle de plantas daninhas em culturas de soja, e principalmente, sua influência sobre a própria cultura, foi conduzido um experimento de campo em $1974 / 75$, no município de Cristais Paulista, SP. O ensaio compunha-se de dez tratamentos distribuídos em blocos ao acaso, com quatro repetições, onde o dinitramine foi aplicado nas doses de $0,375 \mathrm{~kg}$ e $0,575 \mathrm{~kg} / \mathrm{ha}$. Foram plantadas sementes dos cultivares Santa Rosa, IAC-2 e Mineira. Além das testemunhas sem herbicida constou do ensaio um tratamento com trifluralin a $0,720 \mathrm{~kg} / \mathrm{ha}$, plantado com o cultivar IAC- 2 . Em sub-parcelas havia diferencas de profundidade de incorporação dos herbicidas ao solo, a $0,07 \mathrm{~m}$ e $0,15 \mathrm{~m}$.

A população natural de plantas daninhas da área do experimento era representada por duas monocotiledõneas e duas dicotiledôneas, além de outras em pequeno número. As monocotiledõneas foram muito bem controladas por dinitramine nas duas doses e uma das dicotiledôneas também foi eficientemente controlada, porém, somente na dose maior. Trifluralin também deixou de controlar somente uma dicotiledõnea.

A análise estatística do número de plantas de soja revelou diferenças significativas para dinitramine já em sua menor dose, para as três variedades de soja; porém, essa significãncia desapareceu na produção. Por outro lado verificou-se significãncia para a interação tratamento $\mathrm{x}$ incorporação, a qual foi detectado ser o resultado positivo da incorporação rasa com a dose menor de dinitramine.

UNITERMOS: Herbicida, dinitramine, soja.
\end{abstract}

\section{SUM MARY}

EFFECT OF DINITRAMINE, PRE-PLANTING INCORPORATED, ON SOYBEAN CROP (Glycine $\max$ L. MERR.).

\section{L.S.P. CRUZ* E D.A. OLIVEIRA**}

* Seção de Herbicidas

** Seção de Bioestatística. Pesquisadores Científicos, Instituto Biológico, Caixa Postal 70, 13100 - Campinas, SP. Trabalho apresentado na 27. ${ }^{\mathrm{a}}$ Reunião Anual da SBPC realizada em Belo Horizonte, MG, em julho de 1975.

Recebido para publicação em 05.10.79.

They efficiency of dinitramine on weed control and its effects on soybean plants, CV Santa Rosa, IAC-2 and Mineira, was studied in a field trial performed in 1974/75 in Cristais Paulista, SP. Dinitramine was applied at $0,0,375$ and 0,575 $\mathrm{kg} / \mathrm{ha}$, combined with all cultivars, and trifluralin at $0,720 \mathrm{~kg}$, with IAC-2. Plots were split in two incorporation depths: 0,07 and $0,15 \mathrm{~m}$.

Weed population was mainly composed of two monocotyledone and two dycotyledone species. The first were controlled by all treatments and these only with the higher rate of dinitramine. Trifluralin did not control one of the broadleaves.

There were significant reduction for stand by dinitramine for all cultivars, but yields were not reduced. For herbicide $\mathrm{x}$ incorporation depth interaction, the shallower incorporation was best with the smaller rate of dinitramine.

KEYWORDS: herbicides, dinitramine, Glycine mar, incorporation depth.

\section{INT ROD UÇÃO}

Muzik (12) considera que as plantas de crescimento rápido são mais suceptiveis a mostrarem sinais de fitotoxicidade quando submetidas a tratamentos com herbicidas. Para Miyasaká (11), a soja em seus diferentes cultivares indicados para o Brasil, apresenta ciclo cultural variável, bem como variáveis respostas à competição do mato e ao efeito dos herbicidas. Evidentemente, as afirmações dos citados autores complementam-se possibilitando o estabelecimento da hipótese de que a soja, pelo menos em alguns de seus cultivares de menor ciclo, tem condições propícias 
de apresentar sinais de fitotoxicidade quando cultivadas em solos tratados com herbicidas.

Recentemente foi introduzido no Brasil o herbicida dinitramine, o qual pode ser utilizado em diferentes culturas, principalmente na do algodão e da soja $(1,2,3,4,6,8,10,13,15,16)$. A literatura apresenta, com relação a este herbicida e seu uso na cultura da soja, diversos trabalhos de interesse para sua avaliação. Estes trabalhos, referentes a danos à fase cultural e à colheita, apresentam os efeitos não só da dose empregada, bem como do modo de incorporação, sendo que esta é indispensável em virtude da decomposição do produto pela ação da luz solar.

Os trabalhos iniciais datam de 1971, quando Schrades (15) verificou que o dinitramine apresentava efeitos prejudiciais à cultura da soja, na dose de $0,56 \mathrm{~kg}$ a $1,12 \mathrm{~kg} / \mathrm{ha}$, em solos com teor de matéria orgânica variando de 1,2 a 9,0\%. Harvey (4), em 1973, verific ou que doses de dinitramine maiores do que $1,12 \mathrm{~kg} / \mathrm{ha}$ provocavam redução no stand.

O presente trabalho, tendo por finalidade contribuir para o conhecimento da ação do dinitramine sobre a cultura da soja, foi conduzido em 1974, em uma localidade, considerando-se no seu planejamento os problemas referentes às variedades, doses e profundidade de incorporação, os quais, conforme a revisão de literatura realizada, são os mais importantes.

\section{MATERIAL E MÉTODOS}

O experimento foi instalado em 19.11.74 em área de solo latosol vermelho amarelo - fase arenosa, com 30\% de argila e 2,4\% de matéria orgânica. $\mathrm{Na}$ mesma área vinha sendo plantado soja e milho em rotação anual.

O delineamento experimental básico foi o de blocos ao acaso com subdivisão de parcelas para efeito de incorporação dos herbicidas. As parcelas apresentavam 5,OOm de largura e 7,0Om de comprimento, espaçamento de $0,50 \mathrm{~m}$ entre linhas e subparcelas de 2,50 x $7,00 \mathrm{~m}$. As três linhas cen trais de cada subparcela constituíram a parte útil. Foram feitas quatro repetições, dez tratamentos e dois tipos de incorporação. Além dos

*) Usado na formulaçáo comercial de Cobex, concentrado emulsionável com $25 \%$ de dinitramine.

Usado na formulaçấo comercial de Treflan, concentrado emulsionável com $44,5 \%$ de trifluralin. tratamentos principais constituídos em um fatorial de três cultivares de soja e três doses de dinitramine, houve um constituído pelo herbicida trifluralin, usado como padrão, em uma única dose e com um único cultivar de soja, o IAC-2. Os cultivares utilizados dentro do fatorial foram Santa Rosa, Mineira e IAC-2 os quais eram, na ocasião, os mais indicados para o Estado de São Paulo e que, segundo Kiihl e Miyasaka (7), apresentam ciclo vegetativo de 145,160 e 150 dias, respectivamente.

$\mathrm{O}$ dinitramine (N",N"-dietil 2,4-dinitro-6,-trifluorometil-m-fenilone-diamine) (*) foi aplicado nas doses $0,000 \mathrm{~kg}, 0,375 \mathrm{~kg}$ e $0,575 \mathrm{~kg} / \mathrm{ha}$. A aplicação foi feita em pré-plantio da cultura e préemergência do mato, com pulverizador costal, munido de bico de jato em leque 80.03, com um gasto de calda de 400 1/ha. As condições de aplicação do trifluralin $\left({ }^{* *}\right)$ foram semelhantes e a dose utilizada foi de $0,720 \mathrm{~kg} / \mathrm{ha}$. Imediatamente após a aplicação dos herbicidas, procedeu-se sua incor poração ao solo com o emprego de grade de discos, à profundidade de $0,07 \mathrm{~m}$ e de $0,15 \mathrm{~m}$, constituindo assim as sub-parcelas.

A semeadura, na base de 30 sementes inoculadas por metro linear ocorreu na mesma data da incorporação do produto. A adubação foi realizada conforme indicações baseadas na análise de solo, e durante todo o ciclo da soja foi seguido programa preventivo de combate às pragas, igual em todas as parcelas.

A avaliação do efeito dos produtos no controle das principais plantas daninhas foi realizada em $5 \%$ da parcela, segundo preconiza Igue et al. (5). As contagens de plantas daninhas foram feitas 30 dias após a aplicação. De outra parte, procedeu-se a observações visuais constantes, relacionadas à fitotoxicidade dos produtos à cultura da soja, além do efeito destas no stand. Finalmente, procedeu-se a colheita das áreas úteis.

\section{RESULTADOS E DISCUSSÃO}

Controle de plantas daninhas: O quadro 1 apresenta o número das principais plantas daninhas encontradas nos diversos tratamentos componentes do experimento, constituídos pelas monocotiledônea capim - de - colchão - Digitaria sanguinalis (L.) Scop, e capim-péde-galinha - Eleusine indica (L.) Gaertn e pelas dicotiledôneas picão preto - Bidens pilosa L. e poaia branca - Richardia brasiliensis Gomez.

A análise da variância para incidência de plantas daninhas mostrou interações significativas entre tratamen tos e profundidades, não existência de efeitos significativos entre produtos e diferenças marcantes entre as médias destas e das testemunhas. 
Quadro 1 - Número de plantas daninhas por familia na amostragem de 18.12.74.

\begin{tabular}{|c|c|c|c|c|c|c|c|c|c|c|c|c|c|c|c|c|c|c|}
\hline \multirow{3}{*}{ Cultivar } & \multirow{3}{*}{$\begin{array}{c}\text { Dinitramine } \\
\mathrm{kg} / \mathrm{ha}\end{array}$} & \multirow{3}{*}{$\begin{array}{l}\text { Blocos } \\
\text { Incorp. } \\
\text { Famflias }\end{array}$} & \multicolumn{4}{|c|}{1} & \multicolumn{4}{|c|}{2} & \multicolumn{4}{|c|}{3} & \multicolumn{4}{|c|}{4} \\
\hline & & & \multicolumn{2}{|c|}{$\mathbf{P}$} & \multicolumn{2}{|c|}{$\mathbf{R}$} & \multicolumn{2}{|c|}{$\mathbf{P}$} & \multicolumn{2}{|c|}{$\mathbf{R}$} & \multicolumn{2}{|c|}{$\mathbf{P}$} & \multicolumn{2}{|c|}{$\mathbf{R}$} & \multicolumn{2}{|c|}{$\mathbf{P}$} & \multicolumn{2}{|c|}{$\mathbf{R}$} \\
\hline & & & M & D & $\mathbf{M}$ & D & $\mathbf{M}$ & D & $\mathbf{M}$ & D & M & D & $\mathbf{M}$ & D & $\mathbf{M}$ & D & $\mathbf{M}$ & D \\
\hline Santa Rosa & $\begin{array}{l}0,000 \\
0,375 \\
0,575\end{array}$ & & $\begin{array}{r}156 \\
12 \\
19\end{array}$ & $\begin{array}{r}13 \\
15 \\
2\end{array}$ & $\begin{array}{r}151 \\
28 \\
17\end{array}$ & $\begin{array}{r}18 \\
6 \\
10\end{array}$ & $\begin{array}{r}77 \\
5 \\
4\end{array}$ & $\begin{array}{r}41 \\
11 \\
2\end{array}$ & $\begin{array}{r}65 \\
1 \\
10\end{array}$ & $\begin{array}{r}16 \\
3 \\
9\end{array}$ & $\begin{array}{r}52 \\
11 \\
7\end{array}$ & $\begin{array}{l}24 \\
19 \\
18\end{array}$ & $\begin{array}{r}67 \\
17 \\
5\end{array}$ & $\begin{array}{l}26 \\
13 \\
26\end{array}$ & $\begin{array}{r}69 \\
9 \\
25\end{array}$ & $\begin{array}{l}38 \\
23 \\
21\end{array}$ & $\begin{array}{r}90 \\
22 \\
3\end{array}$ & $\begin{array}{r}20 \\
3 \\
7\end{array}$ \\
\hline Mineira & $\begin{array}{l}0,000 \\
0,375 \\
0,575\end{array}$ & & $\begin{array}{r}147 \\
17 \\
46\end{array}$ & $\begin{array}{l}9 \\
8 \\
3\end{array}$ & $\begin{array}{r}132 \\
12 \\
16\end{array}$ & $\begin{array}{l}5 \\
3 \\
7\end{array}$ & $\begin{array}{r}90 \\
2 \\
11\end{array}$ & $\begin{array}{r}5 \\
13 \\
4\end{array}$ & $\begin{array}{r}93 \\
3 \\
5\end{array}$ & $\begin{array}{r}9 \\
16 \\
10\end{array}$ & $\begin{array}{r}76 \\
2 \\
2\end{array}$ & $\begin{array}{r}38 \\
3 \\
25\end{array}$ & $\begin{array}{r}52 \\
0 \\
13\end{array}$ & $\begin{array}{r}17 \\
1 \\
35\end{array}$ & $\begin{array}{r}26 \\
16 \\
5\end{array}$ & $\begin{array}{r}28 \\
7 \\
7\end{array}$ & $\begin{array}{l}58 \\
15 \\
14\end{array}$ & $\begin{array}{r}16 \\
1 \\
9\end{array}$ \\
\hline IAC-2 & $\begin{array}{l}0,000 \\
0,375 \\
0,575\end{array}$ & & $\begin{array}{r}224 \\
10 \\
11\end{array}$ & $\begin{array}{r}13 \\
6 \\
2\end{array}$ & $\begin{array}{r}136 \\
23 \\
67\end{array}$ & $\begin{array}{r}18 \\
5 \\
3\end{array}$ & $\begin{array}{r}46 \\
3 \\
2\end{array}$ & $\begin{array}{r}21 \\
14 \\
4\end{array}$ & $\begin{array}{r}95 \\
9 \\
19\end{array}$ & $\begin{array}{l}19 \\
24 \\
10\end{array}$ & $\begin{array}{r}38 \\
10 \\
5\end{array}$ & $\begin{array}{r}28 \\
6 \\
10\end{array}$ & $\begin{array}{r}42 \\
8 \\
29\end{array}$ & $\begin{array}{r}32 \\
7 \\
46\end{array}$ & $\begin{array}{r}122 \\
4 \\
11\end{array}$ & $\begin{array}{r}6 \\
7 \\
12\end{array}$ & $\begin{array}{r}79 \\
9 \\
25\end{array}$ & $\begin{array}{r}11 \\
10 \\
8\end{array}$ \\
\hline & $0,720(1)$ & & 0 & 2 & 14 & 1 & 5 & 4 & 17 & 2 & 3 & 7 & 6 & 2 & 0 & 15 & 3 & 20 \\
\hline
\end{tabular}

P - incorporação profunda; $M$ - monocotiledônea; (1) -- trifluralin

$\mathbf{R}$ - incorporaçăo rasa ; $\quad$ D - dicotiledônea

Quadro 3 - Dados relativos a stand e produção (kg/ha) de grãos de soja.

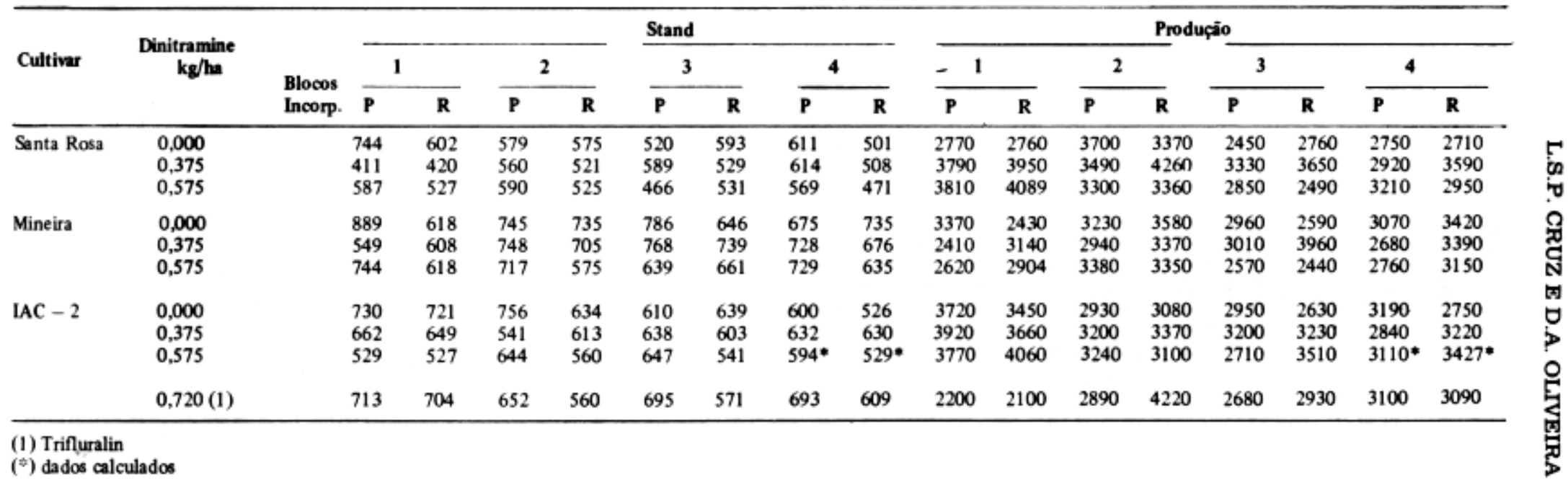


Zuadro 2 - Médias do número de plantas daninhas. Dados em $\sqrt{\mathbf{x}}$.

\begin{tabular}{|c|c|c|c|c|c|c|c|}
\hline \multirow{2}{*}{ Cultivar } & \multirow{2}{*}{$\underset{\mathrm{kg} / \mathrm{ha}}{\text { Dinitramine }}$} & \multicolumn{2}{|c|}{$\mathbf{M}$} & \multicolumn{2}{|c|}{ D } & \multicolumn{2}{|c|}{$M+D$} \\
\hline & & $\mathbf{P}$ & $\mathbf{R}$ & $\mathbf{P}$ & $\mathbf{R}$ & $\mathbf{P}$ & $\mathbf{R}$ \\
\hline \multirow[t]{3}{*}{ Santa Rosa } & 0,000 & 9,2 & 9,5 n.s. & 5,3 & 4,4 n.s. & 10,7 & 10,5 n.s. \\
\hline & 0,375 & 3,0 & 3,8 n.s. & 4,1 & $2,4 *$ & 5,1 & 4,6 n.s. \\
\hline & 0,575 & 3,5 & $2,8 \mathrm{n}-\mathrm{s}$ & 2,9 & 3,5 n.s. & 4,7 & 4,6 n.s. \\
\hline \multirow[t]{3}{*}{ Mineira } & 0,000 & 8,9 & 9,0 n.s. & 4,2 & 3,3 n.s. & 10,1 & 9,7 n.s. \\
\hline & 0,375 & 2,7 & 2,3 n.s. & 2,7 & 1,9 n.s. & 4,0 & 3,3 n.s. \\
\hline & 0,575 & 3,4 & 3,4 n.s. & 2,8 & 3,7 n.s. & 4,9 & 5,1 n.s. \\
\hline \multirow[t]{4}{*}{ IAC-2 } & 0,000 & 9,7 & 9,2 n.s. & 4,0 & 4,4 n.s. & 10,7 & 10,3 n.s. \\
\hline & 0,375 & 2,5 & 3,4 n.s. & 2,8 & 3,2 n.s. & 3,9 & 4,8 n.s. \\
\hline & 0,575 & 2,6 & $5,7^{\circ}$ & 2,5 & 3,6 n.s. & 3,7 & $7,0^{*}$ \\
\hline & $0,720(1)$ & 1,0 & $3,0^{*}$ & 2,5 & 2,1 n.s. & 2,9 & 4,0 n.s. \\
\hline d. m.s & \multicolumn{3}{|c|}{1,7} & \multicolumn{2}{|c|}{1,1} & \multicolumn{2}{|c|}{1,6} \\
\hline
\end{tabular}

(1) trifluralin

Com base no quadro 2, relativo às médias de plantas daninhas, é possivel explicar os pontos fundamentais definidos. Assim é que, com relação à significância das interações, verificou-se para cada família e para o conjunto delas, apenas um contraste significativo para as diferentes profundidades. No caso das monocotiledôneas verificou-se que a incorporação profunda do trifluralin é mais eficiente; e, no caso das dicotiledôneas, o dinitramine a $0,375 \mathrm{~kg} / \mathrm{ha}$ e incorporação profunda para o cultivar Santa Rosa mostrou menor eficiência que a rasa e, finalmente, para as monocotiledôneas e para o conjunto das famílias o dinitramine a $0,575 \mathrm{~kg} / \mathrm{ha}$, na incorporação rasa e cultivar IAC-2, foi menos eficiente que na profunda. Obviamente, em relação a estes resultados apenas o primeiro é racional e explicável, devendo os demais serem computados a ocorrência casuais.

As explicações apresentadas evidenciam que com relação às plantas daninhas não se pode distinguir os diferentes tratamentos com produtos, devendo-se admitir que há um controle efetivo independente de produto, doses de $0,375 \mathrm{~kg}$ e $0,575 \mathrm{~kg} /$ ha e profundidade; concluindo-se que trifluralin e dinitramine foram eficientes no controle das plantas daninhas consideradas, poden- do-se usar a dose de $0,375 \mathrm{~kg} / \mathrm{ha}$ de dinitramine por ser a mais econômica.

Influência no stand: Os resultados relativos a stand estão no quadro 3 . Com base nos resultados apresentados verifica-se que cultivares, doses dos herbicidas e profundidade de incorporação são pontos importantes e que devem ser levados em consideração na apreciação dos produtos. Estas fontes de variação apresentaram efeitos significativos enquanto que suas interações não. Esta ocorrência demonstra a independência entre os fatores e permite que se os analise através de dados médios.

Os dados médios apresentados no quadro 4 demonstram que sob esta característica os três cultivares apresentaram-se com resultados distintos, confirmando pois a existência de respostas diferenciadas ao dinitramine. Por outro lado, não foi notada diferença entre os efeitos deste com os do trifluralin.

No que diz respeito a doses, os resultados são demonstrativos do efeito depressivo do produto no stand, não importando, para a redução, o quanto da dose. A simples presença do produto já o determina. Estes resultados estão conforme o definido anteriormente por Schrader (15), Harvey (4) e Kennedy et al. (6). Estes autores, em seus diferentes trabalhos, utilizaram de doses, em 
termos de princípio ativo, que variavam de $0,55 \mathrm{~kg}$ a $2,24 \mathrm{~kg} / \mathrm{ha}$. Portanto, como no presente trabalho, a dose inferior é de $0,375 \mathrm{~kg} / \mathrm{ha}$, é pertinente considerarse que o limite inferior para danos ao stand ainda não está adequadamente definido. Sob o ponto de vista da profundidade de incorporação, Kennedy et al. (6) apresentaram dados demonstrando a redução do stand com o aprofundamento da incorporação. Os resultados deste trabalho, quadro 4, demonstram o oposto, já que a maior média obtida para stand foi a maiores profundidades. Essa discordância entre resultados não pode ser explicada em termos de herbicida. É mais pertinente considerar-se que o efeito verificado é devido mais à técnica de preparo do solo que propriamente à profundidade de incorporação dos produtos. Esta hipótese é válida já que, conforme o verificado através da não significância das interações a presença ou ausência do produto não modificou a natureza da resposta relacionada ao critério de incorporação. Portanto, é lícito dizer-se que a gradeação rasa para a incorporação do produto, em pré-plantio, prejudicou o stand.

Quadro 4 - Médias para stand, considerando-se os herbicidas, doses utilizadas, cultivar de soja e profundidade de incorporação. Dados em $\sqrt{x}$.

\begin{tabular}{lll}
\hline \multicolumn{1}{c}{ HERBICIDA } & CULTIVAR & MEDIAS \\
\hline Dinitramine & Santa Rosa & $23,3 \mathrm{a}$ \\
& Mineira & $26,3 \mathrm{c}$ \\
& IAC - 2 & $24,8 \mathrm{~b}$ \\
geral & 24,8 \\
Trifluralin & IAC - 2 & 25,5 \\
HERBICIDA & DOSES & \\
Dinitramine & 0,000 & $25,6 \mathrm{~b}$ \\
& 0,375 & $24,7 \mathrm{ab}$ \\
INCORPORAÇÃO & 0,575 & $24,2 \mathrm{a}$ \\
Profunda & & 25,4 \\
Rasa & & 24,4 \\
\hline d.m.s. Tụkey 5\% & & 1,06 \\
\hline
\end{tabular}

Pode-se concluir que a presença de dinitramine, em qualquer das duas doses empregadas, produz redução no stand e que a incorporação profunda, portanto com gradagem a $0,15 \mathrm{~m}$, levou a obtenção do maior stand.

Influência na produção: Os dados de produção são apresentados no quadro 3. Os resultados analíticos evidenciam a capacidade regeneradora da soja, pois, dos efeitos significativos relacionados com o stand para cultivar, dose e profundidade de incorporação, os dois primeiros, justamente os que foram considerados relacionados aos produtos, não apresentaram influência na produção, e o último, mais relacionado ao preparo físico do solo, o foi. Observase ainda que a média geral de produção foi $3151 \mathrm{~kg} / \mathrm{ha}$. Portanto, evidencia-se que o prejuízo ocasionado ao stand não é levado à produção, conforme afirmado também por Harvey (4) e Kennedy et al. (6) em alguns casos.

Por outra parte, o aspecto profundidade de incorporação merece atenção. Notou-se, que em termos médios, a interação incorporação $\mathrm{x}$ tratamento $\mathrm{o}$ foi. Neste particular e a fim de melhor definir as razões desta significância, foi realizada a decomposição possivel para esta interação e verificou-se que todo o efeito é explicado pela interação profundidade de incorporação $\mathrm{x}$ doses. As demais interações não foram significativas, o que implica em dizer que a interação não foi fator preponderante na resposta devida ao cultivar. Nestas condições é válida a avaliação dos resultados sob o ponto de vista de dados médios que englobam os três cultivares. Dentro do observado é indispensável o exame mais detalhado do relacionamento profundidade de incorporação e doses. O quadro 5 resume as informações necessárias. Através desta define-se que na incorporação profunda não houve diferenças significativas entre doses, enquanto que na rasa houve, sendo a dose de $0,375 \mathrm{~kg} / \mathrm{ha}$ a que provocou maior produção, embora a dose 0,575 $\mathrm{kg} /$ ha tenha produzido mais do que a testemunha. Por outro lado, considerando-se as incorporações dentro de doses, encontra-se que na dose de 0,375 $\mathrm{kg} /$ ha a incorporação rasa apresenta maior produção que a profunda, enquanto que nas demais doses não há 
diferenças significativas. Portanto é evidente, no caso em análise, que houve um efeito benéfico provocado pela união incorporação rasa - dose intermediária. É óbvio que esta conclusão é a oposta da ocorrida com o stand, quando a incorporação profunda foi a melhor.

Quadro 5 - Médias de produção de grãos de soja considerando-a em termos de doses e de incorporação, em kg/ha.

\begin{tabular}{lccc}
\hline & \multicolumn{3}{c}{ Doses } \\
\cline { 2 - 4 } Incorporação & $\mathbf{0}$ & $\mathbf{0 , 3 7 5}$ & $\mathbf{0 , 5 7 5}$ \\
\hline Profunda & $3.090,8 \mathrm{a}$ & $3.113,3 \mathrm{a}$ & $3.110,8 \mathrm{a}$ \\
& $\mathrm{a}$ & $\mathrm{a}$ & $\mathrm{a}$ \\
Rasa & $2.956,7 \mathrm{a}$ & $3.563,3 \mathrm{~b}$ & $3.280,1 \mathrm{a}$ \\
& $\mathrm{a}$ & $\mathrm{c}$ & $\mathrm{b}$ \\
\hline
\end{tabular}

d.m.s. para incorporação dentro de dose: 278,8

d.m.s. para dose dentro de incorporaçâo: 437,4

Pode-se concluir, considerando-se a significância definida, que a maior produção, independentemente do cultivar, ocorreu com a incorporação rasa e $0,375 \mathrm{~kg} / \mathrm{ha}$ de dinitramine.

\section{LITERATU RA CITADA}

1. Covolo, L. \& Watt, M.R. Primeiro ensaio de competição der herbicidas na cultura de soja, na regiâo de Santa Maria (RS). In: Sem. Bras. Herb. Ervas Dan., 10. ${ }^{\circ}$ Santa Maria, Resumos, p.60-61, 1974 .

2. Cruz, L.S.P. \& Leiderman, L. Primeiros estudos comparativos do efeito herbicida de fluchloralin e dinitramine para algodoeiro. Bíológico 11: 314-320, 1974.

3. Garcia, I.; Victoria Filho, R. \& Cruz, L.S.P. Controle de plantas daninhas na cultura de algodâo (Gossypium hirsutum L.) com misturas de dinitramine em pré-plantio incorporado. In: Sem. Bras. Herb. Ervas Dan., 11. ${ }^{\circ}$ Londrina. Resumos, p.146, 1976.

4. Harvey, R.G. Comparative study of 12 dinitroaniline herbicides for giant foxtail and velvetleaf control in soybeans. Weed Science 21: $512-516,1973$.
5. Igue, T.; Forster, R. \& Deuber, R. Amostragem mínima em contagem de ervas em experimentos com herbicidas. In: Sem. Bras. Herb. Ervas Dan., $10 .^{\circ}$, Santa Maria. Resumos, p.54, 1974 .

6. Kennedy, J.M.; Frans, R.E. \& Talbert, R.E. Factors affecting the selectivity of dinitramine in soybeans. Arkansas Farm Research 24(6): $15,1976$.

7. Kilihl, R.A.S. \& Miyasaka, S. Descriçáo das principais variedades de soja em cultivo no Estado de Sáo Paulo. In: Simp. Bras. Soja, 1. Campinas, 1970.

8. Lorenzi, H.J. \& Davis, G.G. Competiçăo de herbicidas na cultura da soja. In: Sem. Bras. Herb. Ervas Dan., $11^{\circ}$, Londrina. Resumos, p.67, 1976 .

9. Maksymowicz, Wm. \& Rieck, C.E. Herbicidal properties of soil aplied USB-3584. In: Proc. $25^{\text {th }}$ Am. Meeting Southern Weed Sc. Soc. Abstract, p.414, 1972.

10. Maksymowicz, Wm.; Rieck, C.E.; Egli, D.B. \& Thompson, L. Jr. Temperatura - dinitroaniline interactions in soybeans. In: Proc. 26th Am. Meeting Southern Weed Sc. Abstract, p.64, 1973.

11. Miyasaka, S. Instruçóes para a cultura da soja. Boletim n. ${ }^{\circ}$ 12, Instituto Agronómico de Campinas. 20p., 1965.

12. Muzik, T.J. Weed biology and control. New York Mc Graw Hill Book, 320pp., 1970.

13. Naish, R.W. \& Upritchard, E.A. Dinitramine a new incorporated herbicide for peas, beans and field brassicas. In: Proc. 27th New Zealand Weed Pest. Control Conf., Nelson. Abstracts, p.109-112, 1974 .

14. Ramos, M.G. Controle químico de invasoras na cultura de soja - 75/76. Comunicado Técnico EMBRAPA, dezembro, 1976, Ponta Grossa. Mimeografado, 25pp., 1976.

15. Schrader, J.W. Performance of sevieral substituted aniline herbicides on cotton, peanuts and soybeans on several organic matter levels. In: Proc. 25th Am. Meeting Southern Weed Sc. Soc. Abstracts, p.39, 1972.

16. Venturella, L.R.C.; Ruckheim Filho, O. \& Davis, G.G. Herbicidas isolados no controle às ervas daninhas da soja (Glycine max (L) Merril). In: Sem. Bras. Herb. Ervas Dan., $11^{\circ}$, Londrina. Resumos, p.71, 1976.

17. Victória Filho, R.; Cruz, L.S.P. \& Garcia, I. Comportamento de misturas de dinitramine $\mathrm{e}$ diuron em pré-plantio incorporado na cultura do algodão (Gossypium hirsutum L). In: Sem. Bras. Herb. Ervas Dan., 11. ${ }^{\circ}$, Londrina. Resumos, p.39, 1976.

18. Victória Filho, R.; Garcia, I. \& Cruz, L.S.P, Controle de plantas daninhas na cultura de soja (Glycine $\max (\mathrm{L})$ Merril) com herbicidas em pré-plantio incorporado. In: Sem. Bras. Herb. Ervas Dan., 11. , Londrina. Resumos, p.73, 1976. 